20. Jaffe, I.Z., and Mendelsohn, M.E. 2005. Angiotensin II and aldosterone regulate gene transcription via functional mineralocortocoid receptors in human coronary artery smooth muscle cells. Circ. Res. doi:10.1161/01.RES.0000159937.05502.d1.

21. Huang, P.L., et al. 1995. Hypertension in mice lacking the gene for endothelial nitric oxide synthase. Nature. 377:239-242.

22. Surks, H.K., et al. 1999. Regulation of myosin phosphatase by a specific interaction with cGMPdependent protein kinase I $\alpha$. Science. 286:1583-1587.

23. Tang, M., et al. 2003. Regulator of G-protein signaling 2 mediates vascular smooth muscle relaxation and blood pressure. Nat. Med. 9:1506-1512.

24. Le, T.H., and Coffman, T.M. 2003. RGS2: a "turnoff” in hypertension. J. Clin. Invest. 111:441-443.
doi:10.1172/JCI200317836.

25. Schlossmann, J., et al. 2000. Regulation of intracellular calcium by a signalling complex of IRAG, $\mathrm{IP}_{3}$ receptor and cGMP kinase I $\beta$. Nature. 404:197-201

26. Geiselhoringer, A., et al. 2004. IRAG is essential for relaxation of receptor-triggered smooth muscle contraction by cGMP kinase. EMBO J. 23:4222-4231.

27. Pfeifer, A., et al. 1998. Defective smooth muscle regulation in cGMP kinase I-deficient mice. $E M B O J$. 17:3045-3051

28. Mendelsohn, M.E. 2005. Molecular mechanisms regulating vascular smooth muscle cell tone and blood pressure [abstract]. Keystone Symposia: The Cellular Biol ogy of Atherosclerosis. Keystone, Colorado, USA.

29. Levy, D., et al. 2000. Evidence for a gene influenc- ing blood pressure on chromosome 17: genome scan linkage results for longitudinal blood pressure phenotypes in subjects from the Framingham Heart Study. Hypertension. 36:477-483.

30. Angius, A., et al. 2002. A new essential hypertension susceptibility locus on chromosome 2p24-p25, detected by genomewide search. Am.J. Hum. Genet. 71:893-905.

31. Krushkal, J., et al. 1999. Genome-wide linkage analyses of systolic blood pressure using highly discordant siblings. Circulation. 99:1407-1410.

32. Fernandez-Fernandez, J.M., et al. 2004. Gain-offunction mutation in the KCNMB1 potassium channel subunit is associated with low prevalence of diastolic hypertension. J. Clin. Invest. 113:1032-1039. doi:10.1172/JCI200420347.

\title{
Ikaros transcription factors: flying between stress and inflammation
}

\author{
George P. Chrousos ${ }^{1,2}$ and Tomoshige Kino²
}

${ }^{1}$ First Department of Pediatrics, University of Athens, Athens, Greece. 2Reproductive Biology and Medicine Branch, National Institute of Child Health and Human Development, NIH, Bethesda, Maryland, USA.

\begin{abstract}
The hypothalamic-pituitary-adrenal axis (a major component of the stress system) and the immune system contribute to the maintenance of homeostasis at rest and during stress. Because of their essential roles for the survival of self and species, the activities of these systems have evolutionarily developed in parallel and are intertwined at many levels. In this issue of the JCI, Ezzat et al. demonstrate that Ikaros, a differentiation factor of leukocyte lineage, also influences the maturation of the fetal pituitary corticotroph and, hence, the secretion of adrenocorticotropic hormone before and after birth (see the related article beginning on page 1021). These results indicate that Ikaros is an ontogenetic and phylogenetic integrator of the stress and immune systems and that abnormalities in its function may produce endocrine and/or immune pathologies.
\end{abstract}

\section{The stress and immune systems influence each other's activity}

The stress and immune systems play essential roles in the maintenance of resting and stress-related homeostasis (1-3). The former regulates behavioral, cardiovascular, metabolic, and immune homeostasis, so that individuals can survive under various stressful conditions. The latter distinguishes between self and non-self and between injurious and noninjurious agents and protects the individual from external and

Nonstandard abbreviations used: ACTH, adrenocorticotrophic hormone; AVP, arginine vasopressin; $\mathrm{CRH}$, corticotropin-releasing hormone; GR, glucocor ticoid receptor; HPA, hypothalamic-pituitary-adrenal; $\alpha$-MSH, $\alpha$-melanocyte-stimulating hormone; POMC, proopiomelanocortin.

Conflict of interest: The authors have declared that no conflict of interest exists.

Citation for this article: J. Clin. Invest. 115:844-848 (2005). doi:10.1172/JCI200524886 internal pathogens by eliminating them via several discrete mechanisms. As these 2 systems have been very important for survival of the self and species, they have evolved in parallel, sharing regulatory and mediatory molecules and mutually influencing each other's activities in both a cell- and a tissueand time-specific manner.

When the stress and immune systems are activated during inflammation, their interaction is particularly evident in the relationship between the HPA axis and the inflammatory/immune reaction (Figure 1 and Table 1). The HPA axis, together with the arousal and autonomic systems, form the stress system. The central components of this system are located in the hypothalamus and brain stem. These centers, the paraventricular nucleus (PVN) and the locus caeruleus/norepinephrine system, receive signals of internal and external changes, respectively, from both the central nervous system and distant organs. The PVN transduces these signals to the pituitary gland by secreting corticotropin-releasing hormone (CRH) and arginine vasopressin (AVP) into the hypophyseal portal system. The pituitary gland, in particular its component corticotroph cells, is synergistically activated by these neuropeptides and secretes adrenocorticotropic hormone (ACTH) into the systemic circulation, stimulating the cortices of the adrenal glands to produce glucocorticoids. These systemic end-effector hormones interact with the ubiquitous glucocorticoid receptors (GRs) in target tissues and alter the biologic activities of the brain and most organs and tissues, including the immune system.

In general, activation of the HPA axis alters the activity of certain important facets of the immune system, mainly through the activity of glucocorticoids and their intracellular signaling system $(2,3)$. These hormones, in fact, exert quite diverse actions, both inhibitory and stimulatory, on nearly all aspects of the immune reaction, including the innate, cellular, and humoral immune response (4). Indeed, glucocorticoids inhibit the production of proinflammatory cytokines as well as other mediators of inflammation, such as histamine, bradykinin, serotonin, and many prostanoids, during both the innate and adaptive immune responses. The same hormones, however, stimulate the production of several antiinflammatory 


\section{Table 1}

Inflammatory stress represents unfolding of 2 major biological programs activated during the inflammatory response

\begin{tabular}{|c|c|}
\hline Sickness syndrome & Classic stress syndrome \\
\hline Anorexia, nausea & Anorexia, stimulation of appetite ${ }^{A}$ \\
\hline Fatigue and/or depressed affect & Motivation and/or stimulated affect \\
\hline Somnolence & Arousal \\
\hline Hyperalgesia with or without headache & Analgesia \\
\hline Elevated temperature, fever & Pyrexia, antipyresis ${ }^{A}$ \\
\hline Increased metabolic rate & Increased metabolic rate, return to normal ${ }^{A}$ \\
\hline Acute phase reaction +++++ & Acute phase reaction + \\
\hline \multicolumn{2}{|l|}{ Molecular effectors } \\
\hline $\begin{array}{l}\text { Inflammatory cytokines and/or } \\
\text { mediators, immune } \mathrm{CRH}\end{array}$ & $\mathrm{CRH}, \mathrm{AVP}$, glucocorticoids, catecholamines, immune $\mathrm{CRH}$ \\
\hline \multicolumn{2}{|l|}{ Transcription factors } \\
\hline GR, NF-KB, CREB, Ikaros & GR, NF-кB, CREB, Ikaros \\
\hline
\end{tabular}

cytokines and other mediators, providing antiinflammatory control during an inflammatory reaction and shifting the Th1/Th2 balance toward the Th2 phenotype, which potentiates humoral immunity (4). Glucocorticoids also increase blood neutrophil concentrations and cause a profound depletion of eosinophils and basophils from the circulation. It is fair to summarize that the HPA axis, and glucocorticoids in particular, suppress innate and cellular immunity and promote humoral immunity.

In contrast to the generally suppressive action of the HPA axis on the immune system, the latter mainly activates the former, via several circulating proinflammatory cytokines and other inflammatory mediators and via the afferent sensory and autonomic systems (1-3) (Figure 1). Indeed, TNF- $\alpha$ and IL-1, -6 , and -8 , secreted from local inflammatory sites, independently and synergistically activate the HPA axis by acting on its hypothalamic, pituitary, and adrenocortical components. However, several proinflammatory cytokines, such as TNF- $\alpha$, inhibit the action of glucocorticoids at the level of target tissues by interfering with their intracellular signaling system (4).

\section{Figure 1}

Organization and mutual interactions of the HPA axis and the immune system. Positive and negative influences inside either system are shown as solid and dashed arrows, respectively. Ikaros is expressed and plays an ontogenetic and functional role in both systems. CVO, circumventricular organs; TLR, Toll-like receptor.

\section{The lkaros family of transcription} factors in immune and pituitary cells

Members of the Ikaros family of transcription factors play major roles in the differentiation and biology of leukocytes, including all classes of lymphocytes (NK, $\mathrm{T}$, and $\mathrm{B}$ cells), monocytes/macrophages, and DCs (Figure 2) (5-7). Ikaros was also recently shown to regulate early neutrophil differentiation (8). Thus, Ikaros appears to be a major determinant of immune system development and function. This has been confirmed in studies of homozygous mice with Ikaros gene inactivation, which develop dramatic defects in their lymphoid compartments; $\gamma$-irradiated mice with inactivated Ikaros, which develop thymic lymphomas; and mice in which the Ikaros gene has been inactivated by gene targeting, which develop lymphomas (reviewed in refs. 5-7). The disruption of normal Ikaros function in human infant acute lymphoblastic leukemia, a rare malignancy, further supports the link between Ikaros and immune system development.

Ikaros isoforms share a common C-terminal part, containing a bipartite transactivation domain and 2 zinc finger motifs, which mediate homo- or heterodimerization between isoforms and interaction with other molecules (Figure 3) (5-7). Ikaros isoforms also share a variable $\mathrm{N}$-terminal

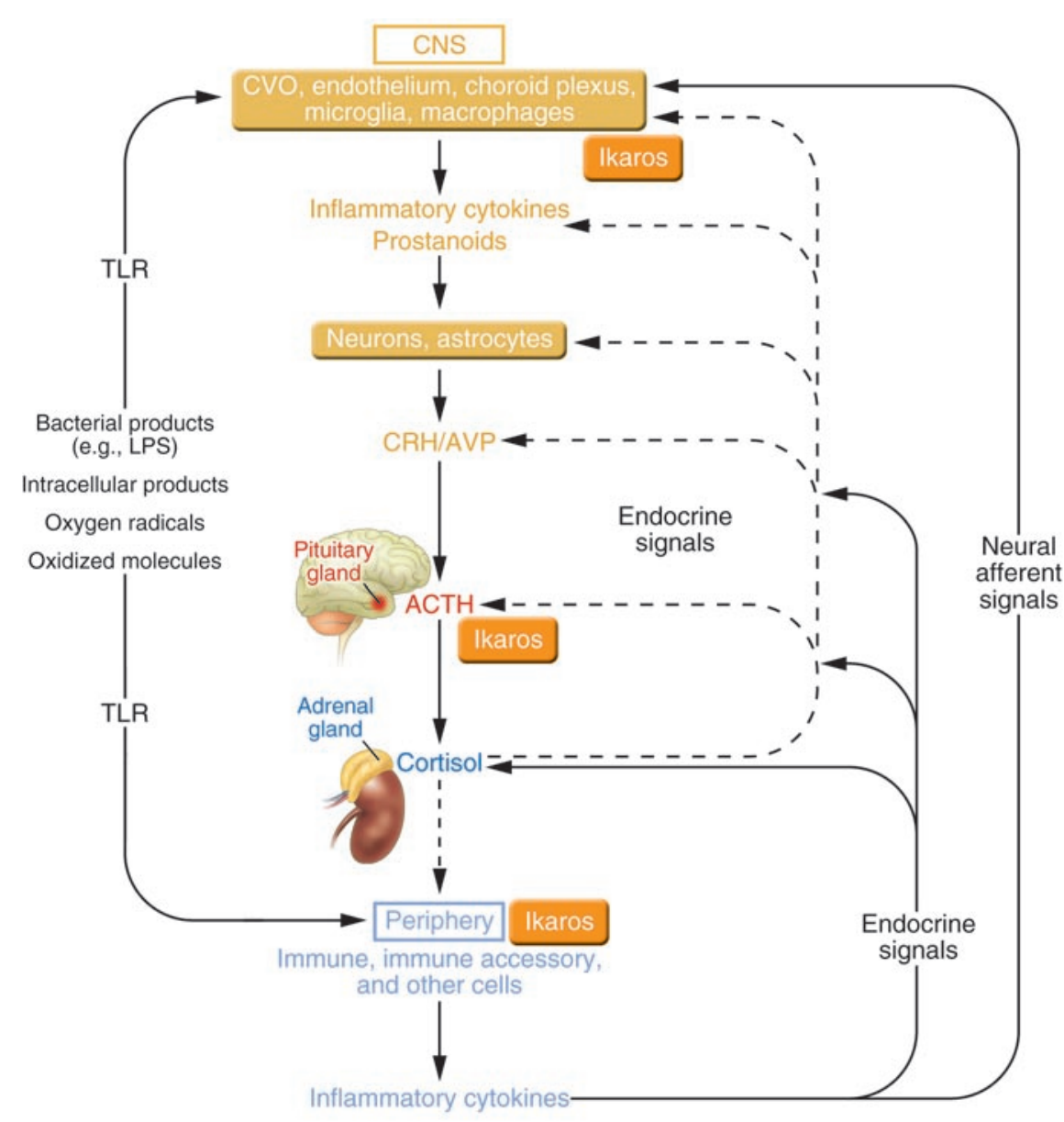


A

Anterior pituitary gland development

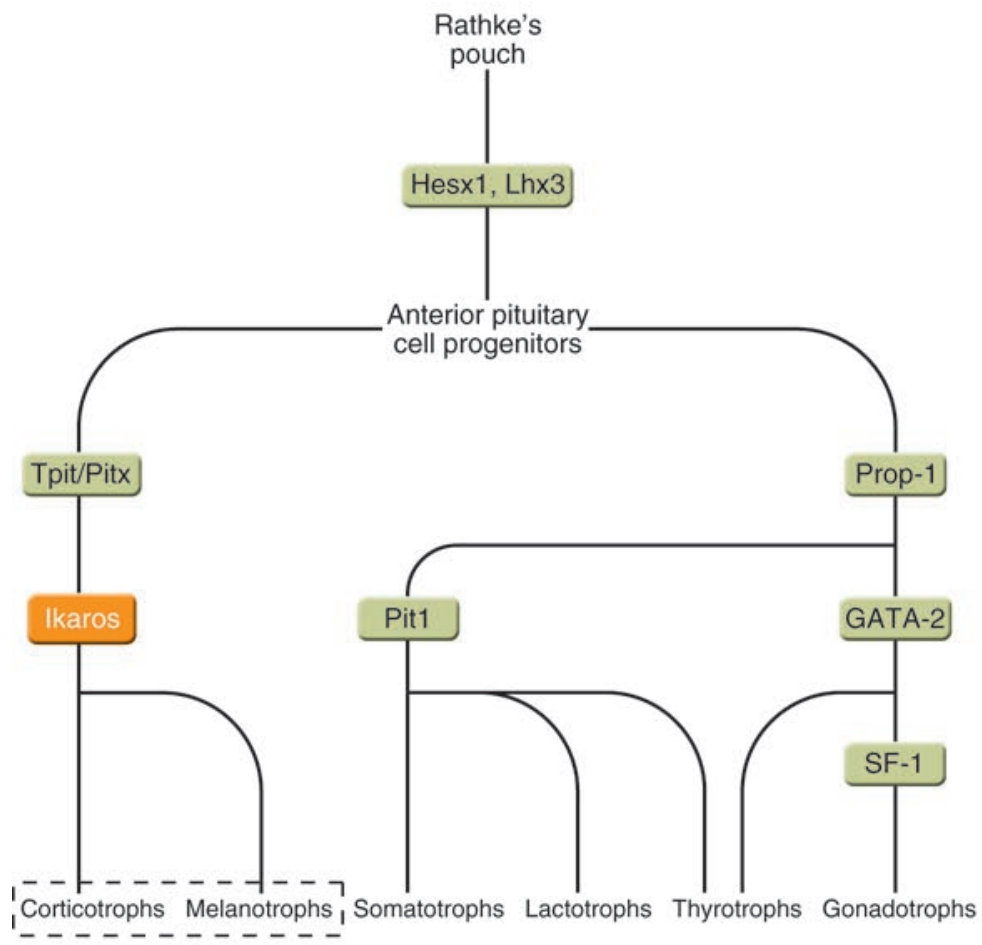

B

Hematolymphopoietic development

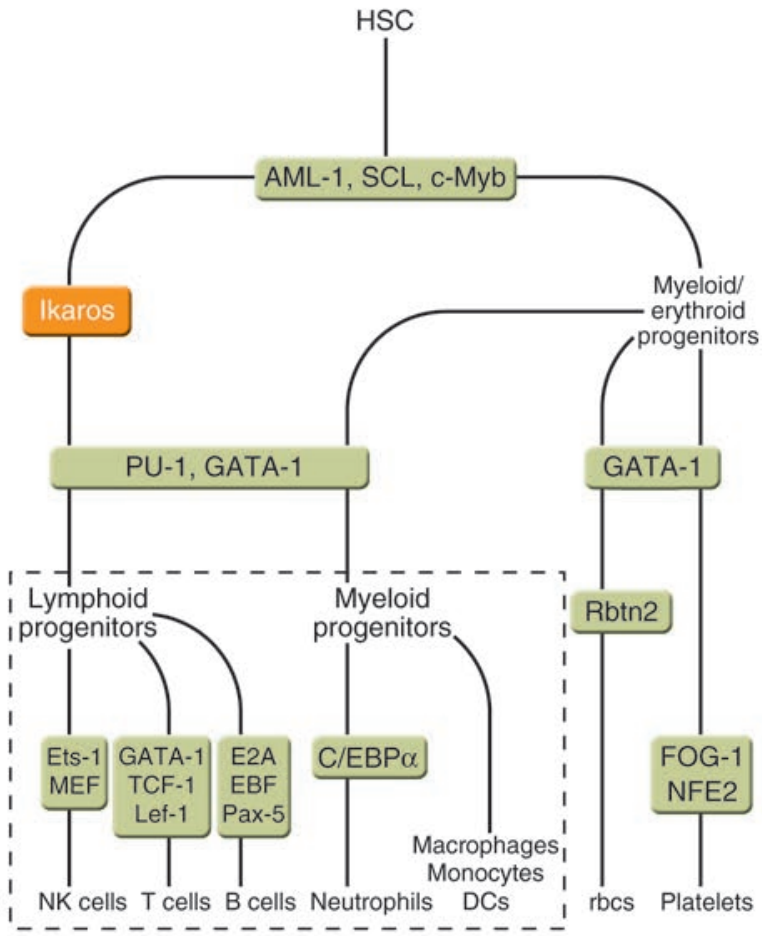

Figure 2

Contribution of Ikaros to the development of the pituitary gland (A) and hematolymphopoietic system (B). Ikaros-dependent differentiated cells are indicated in dashed boxes. Note that in contrast to that of animals, the human anterior pituitary gland contains mainly corticotrophs and expresses mostly ACTH. In animals there are also melanotroph cells that process POMC to MSH, which plays a role in skin and hair pigmentation. AML-1, acute myeloid leukemia 1; C/EBP $\alpha, \mathrm{CAAT} /$ enhancer-binding protein $\alpha$; c-Myb, Myb proto-oncogene; E2A, el2 protein; EBF, early B cell factor; Ets-1, E26 avian leukemia oncogene 1, 5' domain; FOG-1, friend of GATA-binding protein 1; GATA-1, GATA-binding protein 1; Hesx1, homeobox expressed in ES cells 1; Lef-1, lymphoid-enhancing factor 1; Lhx3, LIM homeobox 3; MEF, myeloid elf-1-like factor; NFE2, nuclear factor erythroid-derived 2; Pax-5, paired box gene 5; Pit1, POU domain, class 1, transcription factor 1; Pitx, paired-like homeodomain transcription factor; Prop-1, paired like homeodomain factor 1; PU-1, PU-box binding factor 1; Rbtn2, rhombotin-2; SCL, stem cell leukemia; SF-1, steroidogenic factor 1; TCF, T cell factor; Tpit, pituitary cell-restricted T box factor. Figure modified with permission from Cell (6) and Annual Review of Neuroscience (11).

domain containing different numbers of zinc fingers that mediate the DNA-binding activity of these transcription factors. Since Ikaros only exhibits DNA-binding activity when it has 3 or more $\mathrm{N}$-terminal zinc fingers, isoforms with fewer than 3 zinc fingers may heterodimerize with DNA-binding isoforms and function as their dominantnegative inhibitors. It is appropriate to say that Ikaros represents a complex system of transcription factors rather than a single factor, even though Ikaros isoforms are the products of a single gene.

In this issue of the JCI, Ezzat et al. demonstrate that corticomelanotrophs in the anterior pituitary gland express Ikaros (9). They also show that this complex transcription factor stimulates the transcription rate of the POMC gene in the anterior pituitary gland by binding to specific response elements located in its promoter region (Figure 1). Complete ablation of Ikaros in mice by the Ikaros-null mutation resulted in contraction of the pituitary corticomelanotroph population, reduced circulating ACTH levels, and adrenal insufficiency. These results indicate that Ikaros plays a positive role in the maturation of corticomelanotrophs and their production of ACTH and/or $\alpha$-melanocyte stimulating hormone $(\alpha-\mathrm{MSH})$. In the adult human pituitary gland, there are no melanotrophs, and $\alpha-\mathrm{MSH}$ is not produced by the precursor proopiomelanocortin (POMC) due to different processing of the precursor molecule (Figure 2). Ikaros appears to act downstream of Tpit, a T-box transcription factor that also plays an essential role in corticomelanotroph differentiation and stimulates the transcription rate of the
POMC gene in cooperation with the Pitx homeoprotein $(10,11)$.

\section{Potential clinical implications}

The findings reported by Ezzat et al. (9) suggest that Ikaros facilitates the differentiation and maturation of both pituitary corticotrophs and melanotrophs as well as the leukocyte systems in the developing fetus and quite likely does the same in the pituitary gland and immune system stem cells of the differentiated organism (Figure 2). Genetic alterations of the Ikaros gene may therefore influence both the activity of the HPA axis and immune function. Previous studies of Ikaros mutant animals and human subjects have not reported the activity of the HPA axis; however, it is possible that HPA axis dysfunction went unnoticed in these studies. The findings 

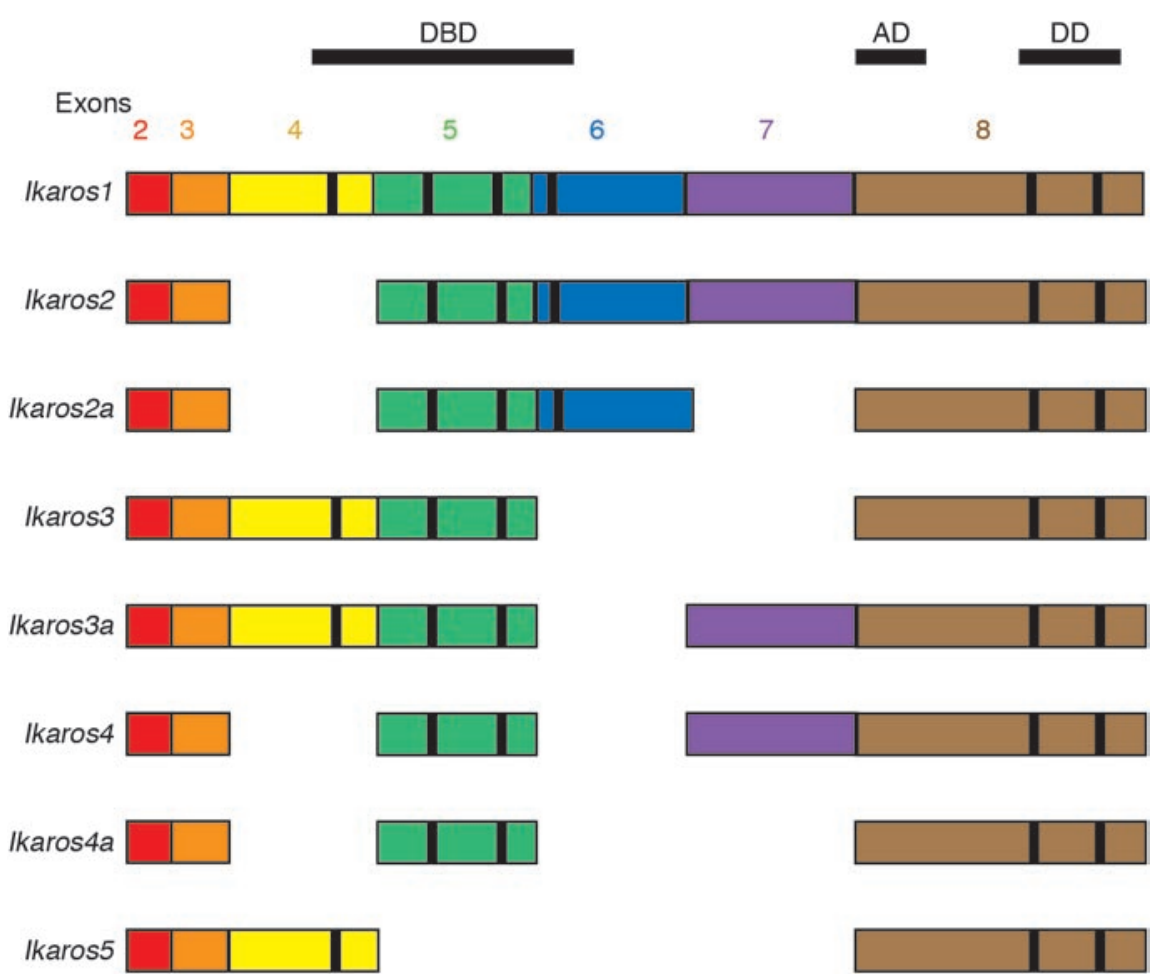

Ikaros6
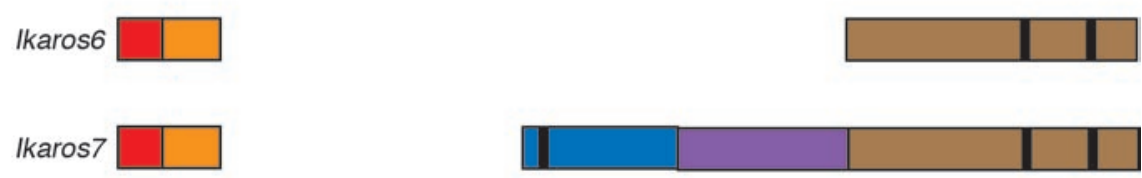

Ikaros8
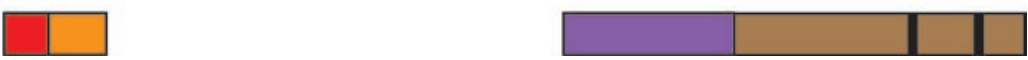

Figure 3

Linearized structure of Ikaros isoforms illustrating distribution of exons, functional domains, and zinc fingers. Zinc fingers are shown as black boxes. Exons 4, 5, 6, and 8 contain 1, 2, 1 , and 2 zinc fingers, respectively. AD, activation domain; DBD, DNA-binding domain; DD, dimerization domain. Figure modified with permission from Immunology and Cell Biology (7) and Trends in Immunology (5).

of Ezzat et al. (9) lead us to hypothesize that the Ikaros gene might be defective in subjects with congenital isolated ACTH deficiency, a disease characterized by low or absent cortisol production secondary to low plasma ACTH despite normal secretion of other pituitary hormones (12). Although Tpit gene mutations account for the development of this rare syndrome in many of the reported cases, some of these patients do not have any defects in this gene. Thus, the presence of this condition in these patients might be related to Ikaros gene dysfunction, a testable hypothesis (Figure 2). At this time, there is no evidence to support or negate the hypothesis that the Ikaros gene is expressed in hypothalamic POMC-manufacturing cells. The main functional products of these cells are $\alpha-\mathrm{MSH}$, which regulates appetite and body weight, and $\beta$-endorphin, which regulates affect and pain sensation. Indeed, if hypothalamic $\alpha$-MSH and $\beta$-endorphin were regulated by Ikaros, one would expect subjects with low Ikaros expression to experience, chronically increased appetite and decreased metabolic rate, leading to obesity and the metabolic syndrome; and defective affect and/or chronic pain and fatigue, leading, respectively, to depression and/or the chronic fatigue and fibromyalgia syndromes.

Changes in Ikaros activity might also produce subtle alterations of the HPA axis and the immune system, expressed as vulnerabilities to certain disorders rather than the disorders themselves. For instance, Fischer and Lewis rats, 2 highly inbred strains, respectively demonstrate increased and decreased responsiveness of the HPA axis to stress as well as decreased and increased susceptibility to experimentally-induced inflammatory diseases and show concurrent behavioral changes (1-3). Mild genetic alterations of the Ikaros gene, such as those produced by minor mutations and polymorphisms, might underlie altered functions of the HPA axis and the immune system, as frequently observed together in experimental animals and humans, and may predispose individuals to disorders related to stress and/or the immune response.

Interaction between Ikaros and the GRs might also be implicated in the development of the HPA axis. Exposure of mothers to stress or glucocorticoids during pregnancy may program the fetal HPA axis for a stressful extrauterine environment (13). Thus, female rats exposed to stress or glucocorticoids during pregnancy deliver pups that have a highly reactive HPA axis and express behaviors throughout their extrauterine lives that are characteristic of stress and anxiety (10-13). Since glucocorticoids regulate the transcriptional activity of Ikaros on the granzyme B promoter, possibly through protein-protein interactions between Ikaros and the GR (14), maternal glucocorticoids might influence the development of the HPA axis and the immune system by modulating Ikaros activity through the GR $(13,15)$. Thus, Ikaros might contribute to the transmission of maternal stress or glucocorticoid effect to the developing fetal HPA axis and immune system in utero, an action that could have lifelong consequences for the individual.

We conclude that the findings of Ezzat et al. (9) on the importance of Ikaros in the ontogeny and regulation of ACTH secretion, along with previous reports focusing primarily on the involvement of Ikaros in the immune response $(6,7)$, suggest that changes in this transcription factor system may be associated with human disorders that are related to dysfunction of the stress and immune responses. These could include states of ACTH, HPA axis, and immune-deficiency. On the other hand, given the prodifferentiation and antitumor effects of Ikaros, one would expect the tumorigenesis of, for example, corticotrophinomas, lymphomas, and leukemias from the corticomelanotroph and leukocyte systems. 
Address correspondence to: Tomoshige Kino, Reproductive Biology and Medicine Branch, National Institute of Child Health and Human Development, National Institutes of Health, Building 10, Clinical Research Center, Room 1-3140, 10 Center Drive MSC1109, Bethesda, Maryland 20892-1109, USA. Phone: (301) 496-5800; Fax: (301) 4020884; E-mail: kinot@mail.nih.gov.

1. Chrousos, G.P., and Gold, P.W. 1992. The concepts of stress and stress system disorders. Overview of physical and behavioral homeostasis. JAMA. 267:1244-1252.

2. Chrousos, G.P. 1995. The hypothalamic-pituitaryadrenal axis and immune-mediated inflammation. N. Engl. J. Med. 332:1351-1362.

3. Chrousos, G.P. 2000. The stress response and immune function: clinical implications. The 1999
Novera H. Spector Lecture. Ann. N. Y. Acad. Sci. 917:38-67.

4. Franchimont, D., Kino, T., Galon, J., Meduri, G.U., and Chrousos, G. 2003. Glucocorticoids and Inflammation Revisited: The State of the Art. NIH Clinical Staff Conference. Neuroimmunomodulation. 10:247-260.

5. Liberg, D., Smale, S.T., and Merkenschlager, M. 2003. Upstream of Ikaros. Trends Immunol. 24:567-570.

6. Georgopoulos, K., et al. 1994. The Ikaros gene is required for the development of all lymphoid lineages. Cell. 79:143-156.

7. Rebollo, A., and Schmitt, C. 2003. Ikaros, Aiolos and Helios: transcription regulators and lymphoid malignancies. Immunol. Cell Biol. 81:171-175.

8. Dumortier, A., Kirstetter, P., Kastner, P., and Chan, S. 2003. Ikaros regulates neutrophil differentiation. Blood. 101:2219-2226.

9. Ezzat, S., et al. 2005. Ikaros integrates endocrine and immune system development. J. Clin. Invest. 115:1021-1029. doi:10.1172/JCI200522486.

10. Lamolet, B., et al. 2001. A pituitary cell-restricted
T box factor, Tpit, activates POMC transcription in cooperation with Pitx homeoproteins. Cell. 104:849-859.

11. Dasen, J.S., and Rosenfeld, M.G. 2001. Signaling and transcriptional mechanisms in pituitary development. Annu. Rev. Neurosci. 24:327-355.

12. Vallette-Kasic, S., et al. 2004. Congenital isolated ACTH deficiency, an underestimated cause of neonatal death, explained by Tpit mutations. J. Clin. Endocrinol. Metab. doi:10.1210/jc.2004-1300.

13. Meaney, M.J. 2001. Maternal care, gene expression, and the transmission of individual differences in stress reactivity across generations. Annu. Rev. Neurosci. 24:1161-1192.

14. Wargnier, A., et al. 1998. Down-regulation of human granzyme B expression by glucocorticoids. Dexamethasone inhibits binding to the Ikaros and AP-1 regulatory elements of the granzyme B promoter. J. Biol. Chem. 273:35326-35331.

15. Challis, J.R., and Brooks, A.N. 1989. Maturation and activation of hypothalamic-pituitary adrenal function in fetal sheep. Endocr. Rev. 10:182-204. 